\title{
Binary fraction of magnetic chemically peculiar stars
}

\author{
E. Paunzen \\ Department of Theoretical Physics and Astrophysics, Masaryk University, \\ Brno, Czech Republic, (E-mail: epaunzen@physics.muni.cz)
}

Received: October 31, 2019; Accepted: January 30, 2020

\begin{abstract}
Magnetic chemically peculiar stars of the upper main sequence are slowly rotating early B to early F-type objects with elemental overabundances of several orders of magnitude compared to the Sun. The driving mechanism is diffusion in the calm stellar atmosphere intensified by the presence of a stable organized magnetic field. The binary fraction is vital to understanding the formation and evolution of these objects as well as possible operating angular momentum loss mechanisms. The available literature on the binary fraction is reviewed and a list of needed future working steps is presented.

Key words: binaries: general - stars: chemically peculiar - stars: magnetic fields
\end{abstract}

\section{Introduction}

Chemically peculiar (CP) stars are upper main sequence objects (spectral types early B to early F) whose spectra are characterized by abnormally strong (or weak) absorption lines that indicate peculiar surface elemental abundances. CP stars constitute about $10 \%$ of upper main sequence stars and are commonly subdivided into four classes (Preston, 1974): metallic line (or Am) stars (CP1), magnetic Bp/Ap stars (CP2), HgMn stars (CP3), and He-weak stars (CP4).

The CP2 and CP4 stars are set apart by the presence of stable, globallyorganized magnetic fields with strengths from about $300 \mathrm{G}$ to several tens of $\mathrm{kG}$. CP1 and CP3 stars do not show strong, organized magnetic fields; the presence of weak fields (less than $100 \mathrm{G}$ ) has been claimed but remains controversial. Common to all subgroups is their apparent slow rotation as compared to normal stars of the same spectral types.

In general, the binary fraction among main sequence stars is depending on mass, orbital period and eccentricity (Moe \& Di Stefano, 2017). Different observational methods are sensitive to different types of binary systems. All this has to be taken into account when determining the overall incidence of binary systems in a specific spectral type range. It is also known that single and binary stars evolve differently, which is particularly true for close systems (Iben, 1991).

The investigation of single magnetic $\mathrm{CP}(\mathrm{mCP})$ stars and $\mathrm{mCP}$ stars in binary systems is important to study the formation and evolution of their stable 
magnetic fields and the loss of angular momentum that these objects undergo. For nearly fifty years (Abt \& Snowden, 1973), the binary fraction among magnetic CP stars has been investigated. Published values range from $20 \%$ to about $50 \%$. Here, an overview of the literature and an outlook of what needs to be done is given.

\section{The importance of the binary frequency}

It has been well established that $\mathrm{CP}$ stars rotate on average more slowly than normal stars of the same spectral types, and that this discrepancy is not due to CP stars being rapid rotators seen pole-on (Netopil et al., 2017). It has therefore been suggested that slow rotation (equatorial velocity $<120 \mathrm{kms}^{-1}$ ) is a necessary (but not sufficient) preliminary condition for the development of chemical peculiarities. CP stars do not simply constitute a slowly rotating tail of "normal" A-type stars; instead, their rotational velocities follow a Maxwellian distribution with an average value three to four times lower. Close binaries might play an important role in the loss of angular momentum on the main sequence.

The origin of the magnetic fields in $\mathrm{mCP}$ stars is still a matter of some controversy, the main competing theories (Moss, 2004; Braithwaite \& Spruit, 2004) being the dynamo theory (field generated by dynamo action in the convective core) and the fossil field theory (field is a relic of the "frozen-in" interstellar magnetic field). Another interesting theory was proposed by Ferrario et al. (2009), who suggested a merger scenario: towards the end of the formation process, after the development of a substantial radiative envelope, a small fraction of stars merge. Such late mergers would result in a brief period of strong differential rotation and give rise to large-scale magnetic fields in the radiative envelopes. This could explain the observed small fraction of magnetic stars that increases with mass. Consequently, we would not expect to find a large fraction of close binary systems among mCP stars.

\section{Published binary fractions}

It is important to note that almost no single CP1 stars are known out of a sample of 4300 objects. The situation is similar for CP3 stars: at least 2/3 out of a sample of about 170 objects are in SB2 systems. The literature concerned with the magnetic subgroups is not conclusive and the results depend on the employed methods and the observational material. In the following, an overview of the most comprehensive papers is given.

- Abt \& Snowden (1973): 20\% out of a sample of 45 stars

- Gerbaldi et al. (1985): $\mathbf{4 6 \%}$ out of a sample of 113 stars

- Carrier et al. (2002): $\mathbf{4 3 \%}$ out of a sample of 119 stars 
- Rastegaev et al. (2014): $\mathbf{2 3 \%}$ out of a sample of 273 stars

- Mathys (2017): 51\% out of a sample of 43 stars

These references include SB1, SB2 and wide binary systems. Up to now, no system harbouring an $\mathrm{mCP}$ stars is known with an orbital period less than $1.5 \mathrm{~d}$. The number of known eclipsing binary systems hosting a magnetic CP star is still below five (Skarka et al., 2019). In summary, it can be said that up to $50 \%$ of all $\mathrm{mCP}$ stars are part of a binary system. A modern sound statistical analysis of the orbital periods, mass ratios, and ages is very much needed to test the different theories.

\section{Conclusions and outlook}

The importance of determining the binary fraction of mCP stars has been outlined. A comparison of single and binary objects will help to put tight constraints on the formation and evolution of this star group. In particular, further investigations should be carried out concerning the merging scenario during the end of the pre-main sequence phase and the apparent need for angular momentum loss to enable effective diffusion in the stellar atmospheres.

The currently available investigations are based on widely different observational material and therefore suffer from various biases. To overcome this unsatisfactory situation, the following research items are suggested:

- comprehensive analysis of known binary systems with a magnetic component using archival spectroscopic and photometric data;

- search for eclipsing binary systems with a spotted upper main sequence primary star in CoRoT, Kepler and TESS data;

- search for short orbital period spectroscopic binaries with an mCP star component;

- spectropolarimetric measurements of known binary systems to detect new mCP star components.

This workflow should guarantee a new observational basis for testing and improving the current models.

\section{References}

Abt, H. A. \& Snowden, M. S., The Binary Frequency for AP Stars. 1973, Astrophys. J., Suppl., 25, 137, DOI: 10.1086/190265

Braithwaite, J. \& Spruit, H. C., A fossil origin for the magnetic field in A stars and white dwarfs. 2004, Nature, 431, 819, DOI: 10.1038/nature02934 
Carrier, F., North, P., Udry, S., \& Babel, J., Multiplicity among chemically peculiar stars. II. Cool magnetic Ap stars. 2002, Astron. Astrophys., 394, 151, DOI: 10.1051/0004-6361:20021122

Ferrario, L., Pringle, J. E., Tout, C. A., \& Wickramasinghe, D. T., The origin of magnetism on the upper main sequence. 2009, Mon. Not. R. Astron. Soc., 400, L71, DOI: 10.1111/j.1745-3933.2009.00765.x

Gerbaldi, M., Floquet, M., \& Hauck, B., Frequency of Bp-Ap stars among spectroscopic binaries. 1985, Astron. Astrophys., 146, 341

Iben, Icko, J., Single and Binary Star Evolution. 1991, Astrophys. J., Suppl., 76, 55, DOI: 10.1086/191565

Mathys, G., Ap stars with resolved magnetically split lines: Magnetic field de-

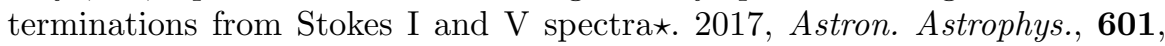
A14, DOI: 10.1051/0004-6361/201628429

Moe, M. \& Di Stefano, R., Mind Your Ps and Qs: The Interrelation between Period (P) and Mass-ratio (Q) Distributions of Binary Stars. 2017, Astrophys. J., Suppl., 230, 15, DOI: 10.3847/1538-4365/aa6fb6

Moss, D., Magnetic fields in A stars. 2004, in IAU Symposium, Vol. 224, The A-Star Puzzle, ed. J. Zverko, J. Ziznovsky, S. J. Adelman, \& W. W. Weiss, 245-252

Netopil, M., Paunzen, E., Hümmerich, S., \& Bernhard, K., An investigation of the rotational properties of magnetic chemically peculiar stars. 2017, Mon. Not. R. Astron. Soc., 468, 2745, DOI: 10.1093/mnras/stx674

Preston, G.W., The chemically peculiar stars of the upper main sequence. 1974, Ann. Rev. Astron. Astrophys., 12, 257, DOI: 10.1146/annurev.aa.12.090174.001353

Rastegaev, D. A., Balega, Y. Y., Dyachenko, V. V., Maksimov, A. F., \& Malogolovets, E. V., Speckle interferometry of magnetic stars with the BTA. II. Results of 2010-2012 observations. 2014, Astrophysical Bulletin, 69, 296, DOI: 10.1134/S1990341314030043

Skarka, M., Kabáth, P., Paunzen, E., et al., HD 99458: First time ever Ap-type star as a $\delta$ Scuti pulsator in a short period eclipsing binary? 2019, Mon. Not. R. Astron. Soc., 487, 4230, DOI: 10.1093/mnras/stz1478 